\title{
La eficiencia de los servicios públicos de empleo en España en un escenario descentralizado: Un análisis desde la perspectiva de la oferta
}

\author{
PATRICIA SUÁREZ CANO \\ Departamento de Economía Aplicada, UNIVERSIDAD DE OVIEDO, ESPAÑA. E-mail: \\ suarezcpatricia@uniovi.es
}

MATÍAS MAYOR FERNÁNDEZ

Departamento de Economía Aplicada, UNIVERSIDAD DE OVIEDO, ESPAÑA. E-mail: mmayorf@uniovi.es

BEGOÑA CUETO IGLESIAS

Departamento de Economía Aplicada, UNIVERSIDAD DE OVIEDO, ESPAÑA. E-mail: bcueto@uniovi.es

\section{RESUMEN}

El principal objetivo del artículo es llevar a cabo un análisis del papel de los Servicios Públicos de Empleo (SPE) en el mercado de trabajo español. Para ello se realiza un análisis a nivel provincial que incorpora diferentes aspectos espaciales así como la estructura descentralizada de las políticas activas de empleo y la existencia de Servicios Públicos de Empleo autonómicos.

La teoría económica señala que, teniendo en cuenta que el objetivo de los Servicios Públicos de Empleo no es tener una alta cuota de mercado en el mercado de trabajo, ésta es mayor en aquellas regiones en las que los mercados de trabajo son menos dinámicos.

Palabras clave: Servicios públicos de empleo, descentralización, autocorrelación espacial.

\section{Efficiency of Public Employment Services in Spain in a Decentralized Framework: An Analysis from the Supply Side}

\begin{abstract}
The article aims to study the role of Public Employment Services (PES) in the Spanish labor market. In order to achieve such an aim, there has been carried out a provincial-level analysis that incorporates several spatial aspects, the decentralization of active labor market policies and the existence of PES dependent on the autonomous communities. Economic theory indicates that the market share of PES is higher in those regions in which the labor market is less dynamic.
\end{abstract}

Keywords: Public Employment Services, Decentralization, Spatial Autocorrelation.

Clasificación JEL: J64, J68, C31

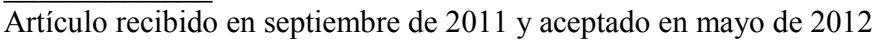

Artículo disponible en versión electrónica en la página www.revista-eea.net, ref. ə-30202 


\section{INTRODUCCIÓN}

En el artículo se pone de manifiesto el desarrollo y la culminación del proceso de transferencia de las políticas activas de empleo centrado en el traspaso a los Servicios Públicos de Empleo autonómicos de la gestión en el ámbito del trabajo, empleo y formación. Por ello, resulta necesario analizar labor realizada por los Servicios Públicos de Empleo desde un punto de vista geográfico-espacial habida cuenta de las diferencias observadas en el análisis de las colocaciones realizado. Las técnicas que nos proporciona la Econometría Espacial resultan adecuadas puesto que nos permiten considerar el papel del espacio en el análisis del mercado de trabajo y más concretamente, en el análisis de los Servicios Públicos de Empleo. La mayor parte de los trabajos realizados hasta el momento ignoran la perspectiva espacial a la hora de analizar las colocaciones en las que intervienen las oficinas de empleo. Con el fin de completar este vacío en la literatura el principal objetivo de este trabajo es analizar la distribución de las colocaciones de los Servicios Públicos de Empleo autonómicos frente al resto de colocaciones y proponer un modelo empírico que explique la cuota de mercado de los SPE autonómicos a nivel provincial.

El artículo se distribuye de la siguiente manera: en el primer apartado se resume la principal bibliografía relacionada con el análisis regional de los Servicios Públicos de Empleo. En el segundo apartado se describen los datos utilizados y se lleva a cabo el análisis espacial de los datos haciendo especial énfasis en la identificación de outliers espaciales, para posteriormente, contrastar la existencia de dependencia espacial global. A partir de estos resultados, en el tercer apartado, se expone la necesidad de utilizar modelos espaciales, que tengan en cuenta explícitamente la dependencia espacial, para explicar el comportamiento del SPE en el mercado de trabajo y se muestran los principales resultados. Finalmente, se recopilan las principales ideas y conclusiones.

\section{ANÁLISIS REGIONAL DE LOS SERVICIOS PÚBLICOS DE EMPLEO}

Si analizamos la literatura existente hasta la fecha sobre los Servicios Públicos de Empleo en España apenas se ha tenido en cuenta la dimensión espacial. Sin embargo, no hay que olvidar que en análisis del mercado de trabajo la dimensión espacial tiene un papel especialmente relevante puesto que empresarios $\mathrm{y}$ trabajadores deben de ponerse en contacto en un lugar y en un momento determinado. La existencia de economías de aglomeración en las principales provincias atrae a los parados que buscan una mayor proximidad geográfica con los empresarios y, por lo tanto, se desplazan hacia lugares donde las oportunidades de encontrar un empleo son mayores. Sin embargo, la presencia de los SPE en su papel de institución que facilita el emparejamiento entre trabajadores y empresarios no solamente en las provincias de referencia, sino también en provin- 
cias con poca población o donde el desarrollo de la actividad económica es menor, contrarresta los efectos derivados de las economías de aglomeración. Russo et al. (2001) muestran para Alemania que es más probable que se use el SPE en aquellos lugares donde existe un exceso de oferta laboral, mientras que cuando existe un exceso de demanda, se utilizan mayoritariamente otros métodos como los anuncios.

En términos más generales, Clinch y O’Neill (2009) subrayan la importancia del espacio a la hora de evaluar el desarrollo de políticas que han supuesto un alto grado de descentralización, siendo fundamental considerar el marco teórico que proporciona la Economía Espacial para llevar a cabo dichas evaluaciones. Por otra parte, existen algunos trabajos que se centran en la labor desarrollada por los servicios públicos de empleo a un nivel regional aunque cada uno de ellos utiliza una metodología diferente. No obstante, el análisis de eficiencia basado en la utilización de fronteras estocásticas o DEA es el más utilizado. Sheldon (2003) y Althin y Behrenz (2004) analizan la eficiencia de los SPE en Suiza y Suecia, respectivamente. En el primer artículo se concluye que la orientación laboral es la medida activa más efectiva e incrementa la eficiencia en el emparejamiento mientras que en el segundo artículo se hace hincapié en la existencia de diferencias en términos de eficiencia a nivel de oficina de empleo. Vassiliev et al. (2006) también evalúan la eficiencia de las oficinas de empleo a nivel local teniendo en cuenta el alto grado de descentralización de las políticas activas de empleo en Suiza.

En Fertig et al. (2006) se analiza si las políticas activas de empleo contribuyen a reducir el desempleo en Alemania a nivel local. Para ello estiman un modelo del retardo espacial (spatial lag) que les permite incluir, a través de la matriz de pesos espaciales utilizada, la existencia de diferentes mercados de trabajo locales en los que actúan las oficinas de empleo. En primer lugar, en este trabajo se menciona la necesidad de que los investigadores puedan acceder a toda la información disponible para lograr un mejor diseño de las políticas de empleo. En segundo lugar se concluye que el reparto de los recursos existentes en cada oficina de empleo entre las posibles medidas activas es una estrategia acertada que redunda en una reducción del nivel de desempleo a nivel local. Además, se señala que los programas de formación son más efectivos que la provisión pública de empleo subvencionado con recursos de las oficinas de empleo.

Aunque no hacen referencia directamente al SPE es necesario destacar también otros estudios que analizan el mercado de trabajo en un contexto espacial como es el caso de Elhorst (2003 y 2008), Elhorst y Zeilstra (2007) y Longhi y Nijkamp (2007). Este último trabajo incorpora las interacciones espaciales en el mercado de trabajo en el análisis del empleo en Alemania y concluye que el marco espacial es especialmente adecuado cuando los datos utilizados hacen 
referencia a unidades administrativas que no necesariamente se corresponden con las áreas naturales de mercado de trabajo. El primer trabajo mencionado de Elhorst proporciona una visión muy completa sobre las explicaciones teóricas y empíricas existentes que explican las diferencias regionales en términos de desempleo. Elhorst y Zeilstra (2007) investiga las diferencias en las tasas de empleo en las regiones europeas e incorpora la existencia de autocorrelación espacial en la modelización. Finalmente en Elhorst (2008) se analizan las tasas de empleo regionales por sexo concluyendo que existen importantes clusters en el espacio.

Karlsson et al. (2002) sintetizan las razones por las cuales debe de contemplarse la perspectiva espacial en el análisis del mercado de trabajo. En primer lugar, el mercado de trabajo no es tan homogéneo como proclamaban las teorías neoclásicas. Es más, desde un punto de vista macroeconómico, la segmentación del mercado de trabajo se asocia con la segmentación espacial. En segundo lugar, se han encontrado patrones espaciales específicos en la distribución del desempleo en varios países. En el caso de España destaca el análisis de la distribución espacial del desempleo que realiza López-Bazo et al. (2002 y 2005) y la aplicación del análisis shift-share espacial sobre el número de ocupados de Mayor y López (2008). Por su parte, Overman y Puga (2002), identifican la existencia de clusters de desempleo en las regiones europeas y consideran que existen disparidades mucho más importantes a nivel regional que a nivel nacional, de manera que no es posible ignorar la perspectiva regional.

Mientras que la literatura internacional es bastante extensa, en España existe un vacío en lo que respecta al análisis, desde un nivel macro, de los Servicios Públicos de Empleo. En Suárez et al. (2011) se analiza el acceso de los parados a las oficinas de empleo teniendo en cuenta la localización de las mismas así como su grado de congestión y si se encuentran ubicadas en municipios urbanos o no. La principal conclusión es que la labor de las oficinas de empleo es especialmente relevante en los municipios no urbanos donde las oportunidades de acceso al empleo son más limitadas. Asimismo, en Suárez et al. (2012) se proponen nuevas medidas de accesibilidad a las oficinas de empleo y se analizan los principales clusters de alta y baja accesibilidad, siendo éstos últimos especialmente relevantes debido a la necesidad de asegurar una adecuada asistencia a los parados en el proceso de búsqueda de empleo. Hay que tener en cuenta que es necesario analizar conjuntamente los Servicios Públicos de Empleo autonómicos sin dejar de lado las particularidades de cada uno de ellos así como su comportamiento diferencial espacialmente.

\section{DATOS}

Los principales usuarios de los SPE son los trabajadores que desean buscar un empleo o mejorar el que ya poseen y que por ello se inscriben en los SPE, es 
decir, son trabajadores que solicitan participar las actividades de intermediación laboral que se llevan a cabo en las oficinas de empleo. Al igual que en otros países europeos, en España no existe la obligación de registrar en las oficinas públicas de empleo los puestos de trabajo que un empresario desea cubrir. No obstante, sí tienen la obligación de comunicar la contratación, de manera que las estadísticas administrativas de contratos recogen este hecho. A continuación, se analiza la Estadística de demandantes de empleo, puestos de trabajo y colocaciones que se constituye como una de las principales fuentes de datos administrativos y que está asociada indisolublemente a la transferencia de la gestión de las políticas activas de empleo a favor de las comunidades autónomas.

\subsection{La estadística de colocaciones}

Las estadísticas derivadas de la implantación del SISPE (Sistema de Información de los Servicios Públicos de Empleo) y concretamente la estadística de colocaciones ha sido muy poco estudiada, con la excepción del trabajo sobre estadísticas administrativas de Toharia (2007) que analiza, entre otros, el fichero de colocaciones registradas en el Servicio Andaluz de Empleo. Las colocaciones son los puestos de trabajo cubiertos por un trabajador, y se asignan a la provincia en que está situado el centro de trabajo. El primer problema que nos planteamos a la hora de seleccionar los datos más apropiados para llevar a cabo este estudio tiene que ver precisamente con la clasificación de las colocaciones o puestos de trabajo cubiertos. Aunque se trata de una estadística difícil de explotar debido a la falta de estudios sobre la misma, consideramos que se trata de la mejor estadística disponible a la hora de evaluar la labor de los SPE.

Al igual que De Koning et al. (1999) hemos adoptado una definición estricta de lo que se considera "mediación del SPE" en la colocación. Así, se definen dos tipos de colocaciones: colocaciones de los Servicios Públicos de Empleo y colocaciones de mercado. Por una parte, las colocaciones de los SPE o puestos de trabajo cubiertos a través del SPE son aquéllos ofrecidos por los empresarios y registrados en las oficinas de empleo públicas con objeto de que éstas gestionen su cobertura con los demandantes más idóneos. Por otra parte, las colocaciones de mercado, son aquellas colocaciones llevadas a cabo sin la intervención de las oficinas de empleo, es decir, ni la oferta estaba registrada en la oficina de empleo ni el trabajador estaba inscrito en la misma.

Se ha utilizado, por lo tanto, la Estadística de colocaciones que proporciona mensualmente datos referentes a las colocaciones para 47 provincias. El periodo de estudio abarca desde enero de 2007 hasta diciembre de 2009 (36 meses) si bien es cierto que se ha calculado el acumulado anual de las categorías estudiadas de manera que trabajaremos con datos anuales referidos a 2007, 2008 y 2009. No obstante, esta agregación temporal ha sido posible gracias a la estabilidad mensual de la cifra de colocaciones (exceptuando los meses de verano) de 
forma que si se trabajase con datos mensuales o trimestrales las conclusiones aquí presentadas no variarían. En la Tabla 1 se muestran los principales estadísticos descriptivos en relación con la variable colocaciones del SPE y de mercado desglosados por sexo.

Tabla 1

Estadísticos descriptivos de las colocaciones

\begin{tabular}{|c|c|c|c|c|c|}
\hline & Año & Desv. & Mín. & Max. & Total \\
\hline \multirow{4}{*}{ Colocaciones SPE Total } & 2007 & $7.224,6$ & 639 & 27.870 & 354.096 \\
\cline { 2 - 6 } & 2008 & $7.072,9$ & 894 & 27.823 & 330.534 \\
\cline { 2 - 6 } & 2009 & $7.455,3$ & 994 & 29.230 & 357.266 \\
\hline \multirow{4}{*}{ Colocaciones de mercado } & 2007 & $362.228,8$ & 21.192 & 1.888 .661 & 12.154 .329 \\
\cline { 2 - 6 } & 2008 & $304.408,3$ & 19.667 & 1.551 .641 & 10.626 .101 \\
\cline { 2 - 6 } & 2009 & $218.150,6$ & 14.190 & 1.069 .964 & 8.188 .825 \\
\hline \multirow{3}{*}{ Colocaciones SPE hombres } & 2007 & $3.178,9$ & 372 & 13.022 & 158.333 \\
\cline { 2 - 6 } & 2008 & $2.791,1$ & 499 & 13.199 & 141.528 \\
\cline { 2 - 6 } & 2009 & $3.312,4$ & 577 & 14.788 & 175.112 \\
\hline \multirow{3}{*}{ Colocaciones de mercado Hombres } & 2007 & $191.124,4$ & 10.729 & 977.375 & 6.802 .932 \\
\cline { 2 - 6 } & 2008 & $156.619,3$ & 9.942 & 788.077 & 5.735 .903 \\
\cline { 2 - 6 } & 2009 & $110.135,3$ & 7.078 & 534.419 & 4.314 .300 \\
\hline \multirow{3}{*}{ Colocaciones SPE mujeres } & 2007 & $4.306,6$ & 267 & 15.728 & 195.763 \\
\cline { 2 - 6 } & 2008 & $4.424,6$ & 395 & 15.619 & 189.006 \\
\cline { 2 - 6 } & 2009 & $4.218,5$ & 407 & 14.442 & 182.154 \\
\hline \multirow{3}{*}{ Colocaciones de mercado Mujeres } & 2007 & $169.614,7$ & 9.451 & 881.701 & 5.351 .397 \\
\cline { 2 - 6 } & 2008 & $149.272,4$ & 9.196 & 763.564 & 4.890 .198 \\
\cline { 2 - 6 } & 2009 & $109.938,0$ & 7.112 & 569.051 & 3.874 .525 \\
\hline
\end{tabular}

Fuente: Elaboración propia a partir de los datos del Servicio Público de Empleo Estatal (SEPE).

Lo primero que hay que destacar es que, en términos generales, a lo largo del periodo 2007-2009 aumentan los valores mínimos y máximos de las colocaciones del SPE mientras que disminuyen los valores mínimos y máximos de las colocaciones de mercado, excepto el máximo de las colocaciones del SPE para las mujeres que no aumenta sino disminuye. En definitiva, con la crisis económica la actividad del SPE en términos de colocaciones ha aumentado cuando la tendencia del resto de colocaciones ha sido la contraria.

\section{2. ¿Cómo se distribuyen las colocaciones en España?}

El presente apartado se centra en el estudio de la distribución de las colocaciones en España. Para ello, en primer lugar se visualiza su distribución espacial, distinguiendo entre colocaciones del SPE y colocaciones de mercado para, en segundo lugar, analizar la importancia de las colocaciones del SPE con respecto a las colocaciones de mercado y ver si existen grandes diferencias en el 
espacio. Antes de pasar al análisis de estos patrones espaciales resulta de gran utilidad analizar los "box-map" de ambos tipos de colocaciones. Este tipo de mapas permite clasificar a las provincias en función del cuartil en el que se encuentran dado su nivel de colocaciones así como identificar las localizaciones atípicas (outliers superiores e inferiores).

Las Figuras 1, 3 y 5 muestran los mapas en los que aparece representada la variable colocaciones del SPE para 2007, 2008 y 2009. De igual forma, las Figuras 2, 4 y 6 muestran los mapas correspondientes a la variable colocaciones de mercado. En este caso, los mapas de cuartiles (cuatro cuantiles) permiten comparar las observaciones atípicas para diferentes variables.

Se observa que en la distribución de ambos tipos de colocaciones no existen lower outliers en ninguno de los años considerados, tal y como es de esperar puesto que los SPE autonómicos así como el SEPE están presentes en todo el territorio nacional garantizando una participación mínima en el ámbito de las colocaciones.

Por el contrario, existen upper outliers no coincidentes en el espacio (con la excepción de Madrid) tanto en el caso de las colocaciones del SPE como para las colocaciones de mercado. Se observa que, en el caso de las colocaciones del SPE, dejando al margen del análisis Madrid, destaca Badajoz en 2007, Cáceres, Badajoz, Sevilla y Córdoba en 2008 y Badajoz, Cáceres, Córdoba, Jaén y Sevi1la, en 2009.

En el caso de las colocaciones de mercado, dejando nuevamente al margen del análisis Madrid, se constituyen como upper outliers, Barcelona, Murcia, Sevilla y Valencia en 2007, Barcelona, Sevilla y Valencia en 2008 y finalmente Barcelona y Valencia en 2009.

Se puede decir de forma genérica que, con el empeoramiento del ciclo económico, la presencia del SPE es mayor mientras que en las colocaciones de mercado ocurre lo contrario. Esta idea refuerza la mayor intervención del SPE en periodos de crisis. Existen trabajos previos que señalan que en momentos de recesión se registra y se cubre un mayor porcentaje de vacantes en las oficinas de empleo. Además, las vacantes registradas sufren una menor fluctuación que el total de vacantes ante una recesión.

Además de las conclusiones anteriores basadas en el análisis de las colocaciones es necesario obtener otros esquemas de visualización en términos relativos, por lo que se propone utilizar como indicador relativo la proporción que suponen las colocaciones del SPE sobre las colocaciones de mercado (Figura 7), distinguiendo entre hombres (Figura 8) y mujeres (Figura 9). En términos generales, se sigue observando que las provincias, especialmente las que se encuentran en el último cuartil, tienden a localizarse juntas. Se observa este hecho en Andalucía, Castilla-La Mancha y Extremadura mientras que en el litoral mediterráneo y en la zona de La Rioja, Navarra y País Vasco, se encuen- 
tran las provincias que se sitúan en los dos primeros cuartiles. Especialmente interesante son los mapas en el caso de las mujeres puesto que ponen de manifiesto un patrón espacial muy significativo en el sur de la península afectando a gran parte de Andalucía, Castilla-La Mancha y Extremadura donde se observan valores más elevados del peso de las colocaciones del SPE respecto a las colocaciones de mercado. En el caso de los hombres no existe un esquema de asociación espacial tan importante aunque, de hecho, se observa que en algunas provincias del norte (Lugo, Orense, León o Zamora, por ejemplo) la actividad del SPE es más elevada que en el sur de la península.

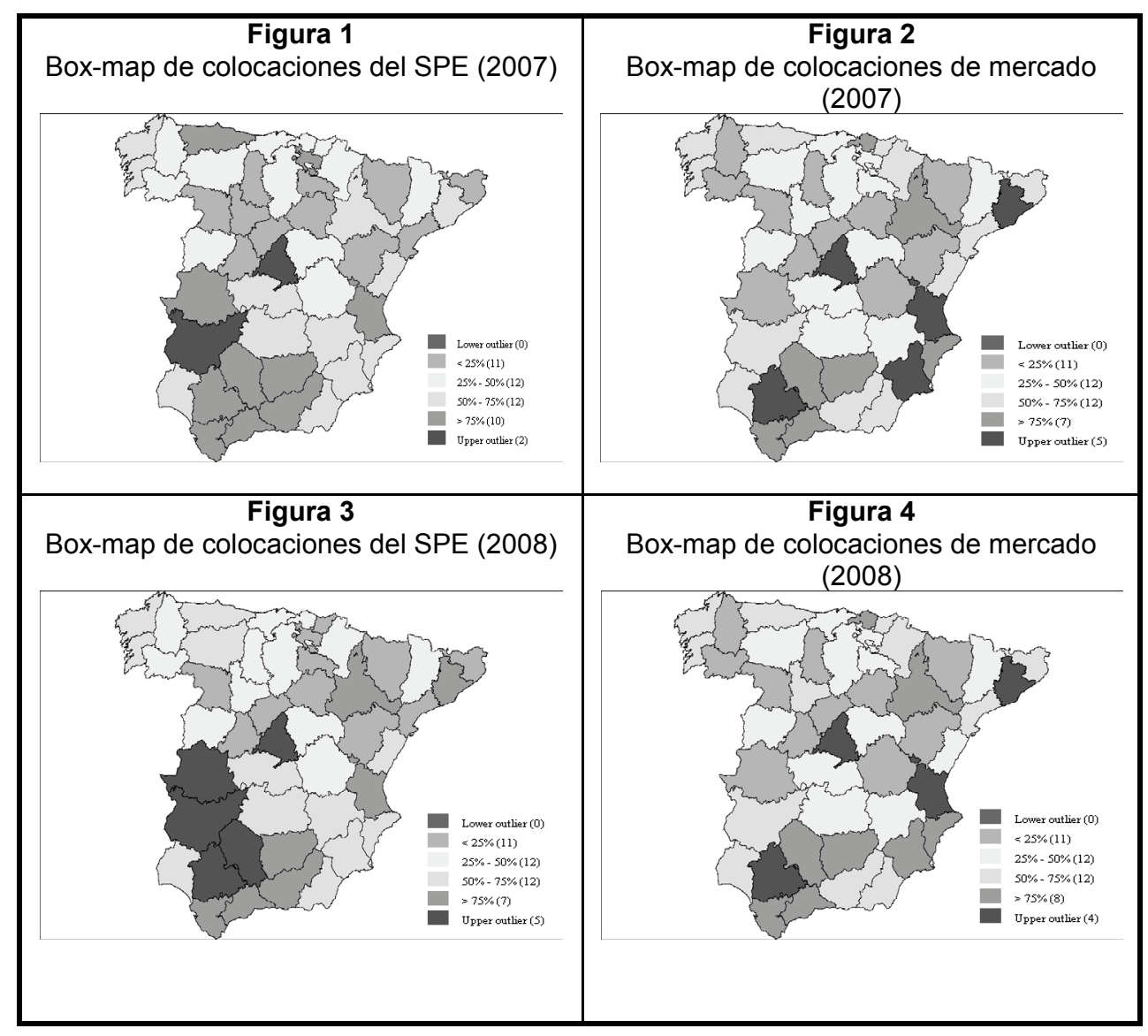




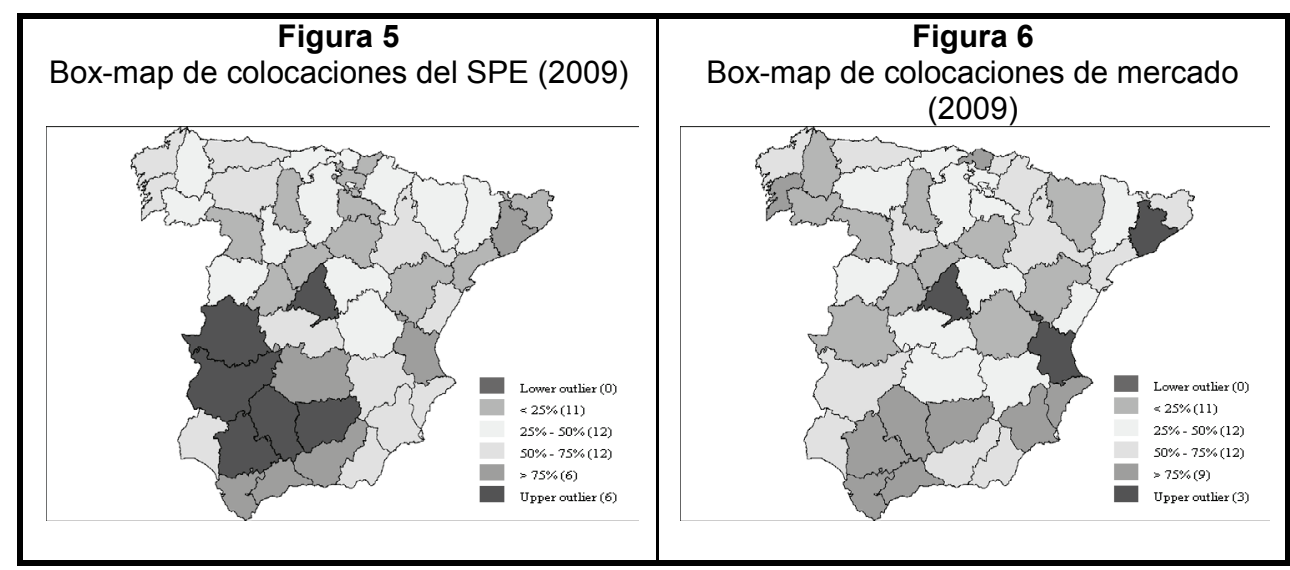

Fuente: Elaboración propia a partir de los datos del SEPE.

Figura 7

Box-map de las colocaciones del SPE sobre las de mercado (ambos sexos)

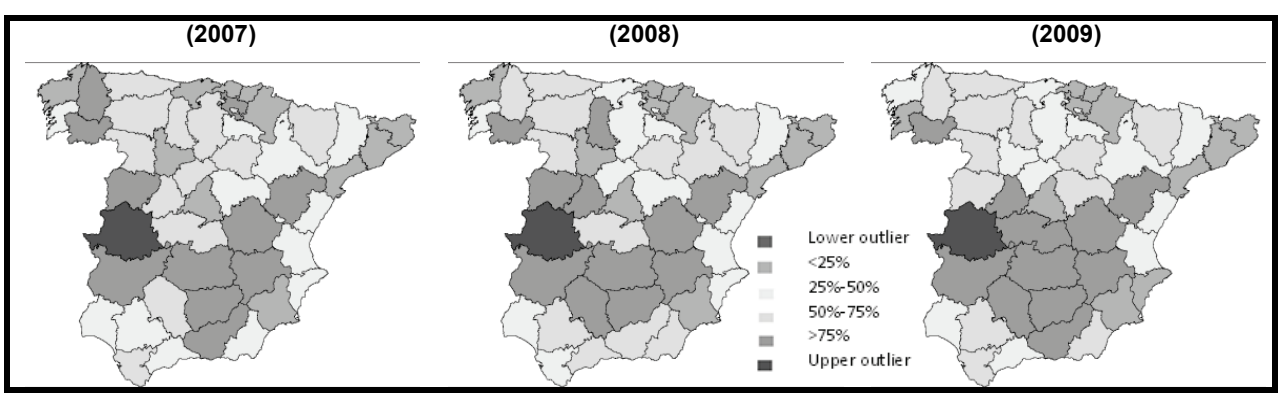

Fuente: Elaboración propia a partir de los datos del SEPE.

Figura 8

Box-map de las colocaciones del SPE sobre las de mercado (hombres)

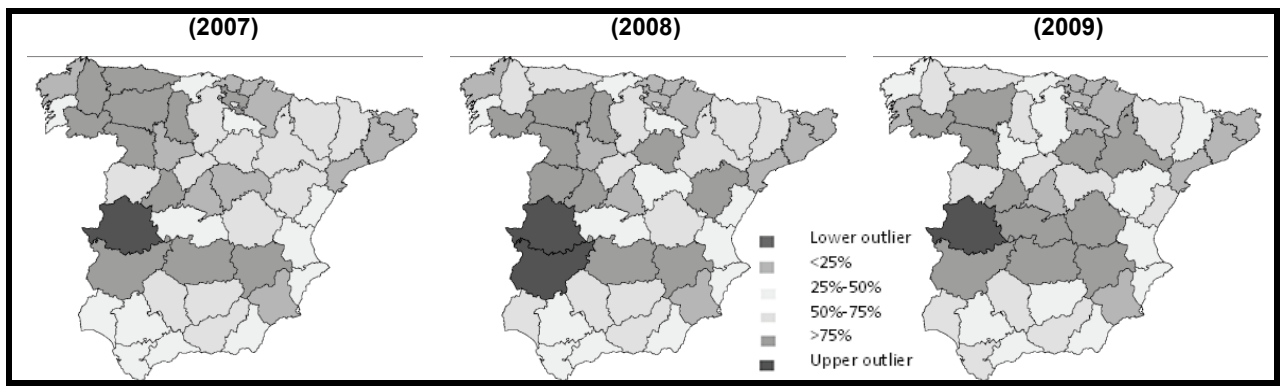

Fuente: Elaboración propia a partir de los datos del SEPE. 
Figura 9

Box-map de las colocaciones del SPE sobre las de mercado (mujeres)

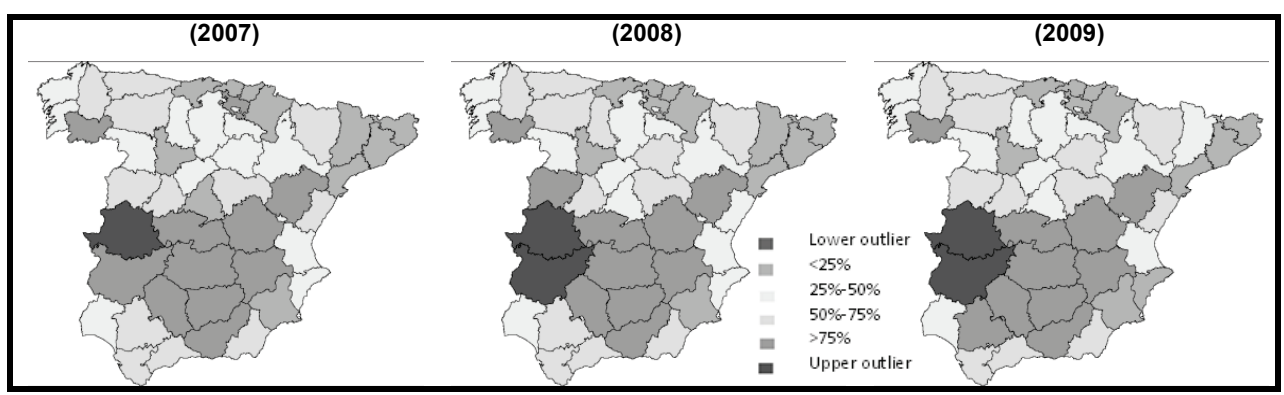

Fuente: Elaboración propia a partir de los datos del SEPE.

La evidencia empírica observada hasta el momento pone de manifiesto dos aspectos clave, por una parte, un comportamiento espacial diferenciado para hombres y mujeres que ha de ser considerado en cualquier intento posterior de modelización y, por otra, que la utilización de técnicas apropiadas permiten la confirmación de la existencia de dichos patrones espaciales y su inclusión explícita en los modelos correspondientes.

\section{MODELIZACIÓN EN PRESENCIA DE DEPENDENCIA ESPACIAL}

Cuando se trabaja con datos de corte transversal es muy habitual encontrarse con un esquema de dependencia espacial. Si el valor de una variable en un lugar está relacionado con el valor de la misma variable en otro lugar o lugares se habla de dependencia o autocorrelación espacial. Estas relaciones espaciales se caracterizan por la multidireccionalidad de manera que es necesario resolver los problemas que ocasiona la presencia de este tipo de efectos espaciales. En los últimos años la econometría espacial ha experimentado un fuerte desarrollo. La mayor parte de los estudios de carácter empírico que utilizan dicha metodología se han vinculado a la economía regional y urbana y en mucha menor medida a la economía laboral. A diferencia de lo que ocurría en el pasado, la existencia de dependencia espacial es más la regla que la excepción, y en lo relacionado con el mercado de trabajo ocurre lo mismo. Hoy en día, existen razones teóricas y empíricas para incorporar este hecho a las investigaciones relacionadas con el mercado de trabajo ${ }^{1}$.

1 Véanse los trabajos de Molho (1995), Longhi y Nijkamp (2007) o Patacchini y Zenou (2007), entre otros. 
A continuación, se definen los conceptos de autocorrelación o dependencia espacial y las matrices de pesos utilizadas, se analizan los resultados del principal test existente para contrastar la existencia de autocorrelación y se describen brevemente los modelos espaciales más habituales con el objetivo de aportar nuevos resultados empíricos sobre la actividad de los SPE autonómicos.

\subsection{Autocorrelación o dependencia espacial y matrices de pesos espaciales}

Cliff y Ord (1973), Paelink y Klaassen (1979) y Anselin (1988) definen la dependencia o autocorrelación espacial como la ausencia de independencia entre las observaciones analizadas o lo que es lo mismo, la existencia de una relación funcional entre lo que ocurre en un determinado punto del espacio y lo que ocurre en otro lugar. En los trabajos empíricos, los conceptos de dependencia y autocorrelación espacial son utilizados indistintamente aunque no son sinónimos, siendo la autocorrelación espacial una expresión más débil de la dependencia espacial. Como trabajo empírico que es, en lo que sigue, ambos conceptos se utilizan indistintamente.

La autocorrelación espacial puede ser positiva o negativa. Existe autocorrelación espacial positiva cuando lo que sucede en un determinado lugar lleva a que se extienda ese mismo fenómeno hacia el resto de lugares que lo rodean, favoreciendo así la concentración del mismo. Por el contrario, existe autocorrelación negativa cuando lo que sucede en un determinado lugar no puede aparecer en los lugares que lo rodean. El ejemplo tradicional de autocorrelación negativa o yuxtaposición de valores es la disposición de las casillas blancas y negras en un tablero de ajedrez. En resumen, la autocorrelación espacial positiva está relacionada con la similitud de valores y la autocorrelación espacial negativa con la disimilitud de valores cercanos.

La multidireccionalidad que caracteriza las relaciones espaciales complica su introducción en los modelos empíricos de manera que una de las opciones para incluirlas en los modelos es utilizar una matriz de pesos espaciales. Existen diferentes criterios para definir la matriz de pesos espaciales y de hecho, no existe acuerdo en la literatura sobre el criterio óptimo de construcción de la matriz. El criterio más utilizado recoge el concepto de contigüidad física, donde $w_{i j}=1$ si las unidades espaciales son físicamente adyacentes mientras que $w_{i j}=0$ en caso contrario.

En este trabajo se ha definido una matriz que recoge por un lado, el concepto de contigüidad física y, por otro, el hecho de que una provincia pertenezca a una determinada comunidad autónoma. La idea que subyace en la consideración conjunta de ambas matrices es que, en la medida en las provincias que pertenecen a una misma comunidad autónoma, comparten un mismo modelo de gestión, pautado por el Servicio Público de Empleo autonómico, de las políticas 
activas de empleo y de las oficinas de empleo que difiere de la gestión y del mayor o menor esfuerzo de otros Servicios Públicos de Empleo autonómicos. Esta consideración afecta directamente al peso que tendrán las colocaciones del SPE sobre las colocaciones de mercado.

A partir de esta matriz se contrasta si existe un patrón espacial en la distribución del peso de las colocaciones del SPE con respecto a las colocaciones de mercado o si por el contrario la distribución es aleatoria. En primer lugar, se calcula el estadístico de Moran $^{2}$ que mide la intensidad de la autocorrelación espacial en la distribución del peso de las colocaciones del SPE sobre las colocaciones de mercado con el fin de corroborar de forma empírica la necesidad de incluir la perspectiva espacial ya apuntada por la teoría económica. Este estadístico se ha calculado para los años 2007, 2008 y 2009 para el peso total y distinguiendo por sexo. En la Tabla 2 se incluyen también los resultados del contraste de significación asociado a la I de Moran obteniéndose evidencia para rechazar la hipótesis nula de no autocorrelación espacial ${ }^{3}$.

Tabla 2

Autocorrelación espacial global (estadístico de Moran)

\begin{tabular}{|c|c|c|c|}
\hline & Moran & Z & Probabilidad \\
\hline Colocaciones SPE/Colocaciones de mercado 2007 & 0,150 & 2,290 & 0,010 \\
\hline C. SPE/C. mercado 2007 (hombres) & 0,216 & 3,436 & 0,000 \\
\hline C. SPE/C. mercado 2007 (mujeres) & 0,108 & 1,575 & 0,057 \\
\hline C. SPE/C. mercado 2008 & 0,205 & 3,810 & 0,000 \\
\hline C. SPE/C. mercado 2008 (hombres) & 0,218 & 4,010 & 0,000 \\
\hline C. SPE/C. mercado 2008 (mujeres) & 0,203 & 3,216 & 0,000 \\
\hline C. SPE/C. mercado 2009 & 0,288 & 4,492 & 0,000 \\
\hline C. SPE/C. mercado 2009 (hombres) & 0,276 & 4,408 & 0,000 \\
\hline C. SPE/C. mercado 2009 (mujeres) & 0,323 & 4,253 & 0,000 \\
\hline
\end{tabular}

Fuente: Elaboración propia.

Conforme a la explicación anterior sobre los diferentes tipos de autocorrelación, se concluye que las variables analizadas muestran autocorrelación espacial

${ }^{2}$ El estadístico de Moran presenta la siguiente expresión: $I=\frac{N}{S_{o}} \frac{\sum_{i j}^{N} w_{i j}\left(x_{i}-\bar{x}\right)\left(x_{j}-\bar{x}\right)}{\sum_{i=j}^{N}\left(x_{i}-\bar{x}\right)^{2}} i \neq j$ donde $x_{i}$ es el valor de la variable $x$ en la región $i, \bar{x}$ es su media, $w_{i j}$ son los pesos de la matriz, $\mathrm{y}$ $S_{o}=\sum_{i j} w_{i j}$.

3 En un documento de trabajo previo a este artículo (Suárez y Mayor, 2009) se muestran los contrastes de autocorrelación local (LISA) sobre las variables colocaciones del SPE y de mercado. 
positiva. Además, se observa como el estadístico de Moran aumenta desde 0,15 en 2007 hasta casi 0,30 en 2009.

\subsection{Los modelos espaciales}

Tomando como referencia el reciente artículo de Elhorst (2010) en el cual se destacan las aportaciones del libro de LeSage y Pace (2009) en el ámbito de la econometría espacial, se procede a comentar, sin ánimo de exhaustividad, los principales modelos de corte transversal que incorporan la existencia de dependencia espacial. El modelo inicial y más completo es el Manski que incorpora tanto la variable dependiente como la matriz de variables explicativas modificadas espacialmente. Asimismo, se recoge también dicha modificación espacial en el término de error. Manski (1993) señala la existencia de tres tipos diferentes de efectos interacción espacial que explican por qué el valor de una variable en una localización específica depende de lo que ocurre en otras localizaciones. El primero es una interacción endógena de forma que el comportamiento de la variable a explicar en una unidad espacial depende del comportamiento de esa misma variable en otras unidades espaciales $(W Y)$, interacciones exógenas, donde el comportamiento de la variable a explicar depende del comportamiento de las variables explicativas en otras unidades espaciales $(W X)$ y por último, los efectos de interacción entre las perturbaciones aleatorias de las diferentes unidades espaciales $(W u)$.

El resto de modelos, que se recogen en la Figura 10, se derivan de este modelo inicial incorporando más o menos restricciones. Los modelos más utilizados son el modelo del retardo espacial (spatial lag) y el modelo del error espacial (spatial error). La principal diferencia entre ambos tipos estriba en la forma en la que se especifica la dependencia espacial, si es la variable dependiente la que se modifica espacialmente (spatial lag) o si la dependencia espacial está incluida en el término de error (spatial error).

Tanto la variable dependiente como las independientes han sido consideradas en términos logarítmicos para facilitar la interpretación de los resultados. En este caso la variable dependiente es el peso de las colocaciones del SPE sobre las colocaciones de mercado y las variables explicativas finalmente incluidas son la tasa de actividad y el salario medio de la provincia. Inicialmente se han considerado otras variables explicativas que han sido descartadas para evitar problemas de endogeneidad como, por ejemplo, el número de puestos registrados en las oficinas de empleo, la tasa de paro o el número de beneficiarios de prestaciones por desempleo. Además, se ha planteado analizar la existencia de efectos producidos por las economías de aglomeración a través de la variable densidad de población si bien los resultados no son significativos. También se ha tratado de incorporar la estructura productiva de las provincias buscando una posible relación entre ésta y el diferente peso provincial de los SPE. Sin em- 
bargo, no se han encontrado resultados concluyentes. Finalmente, dado el diferente comportamiento observado en apartados anteriores al desglosar los resultados por sexo, se ha procedido a estimar los modelos distinguiendo por sexo, siguiendo lo propuesto Elhorst (2007).

Figura 10

Modelización de la dependencia espacial en modelos de corte transversal

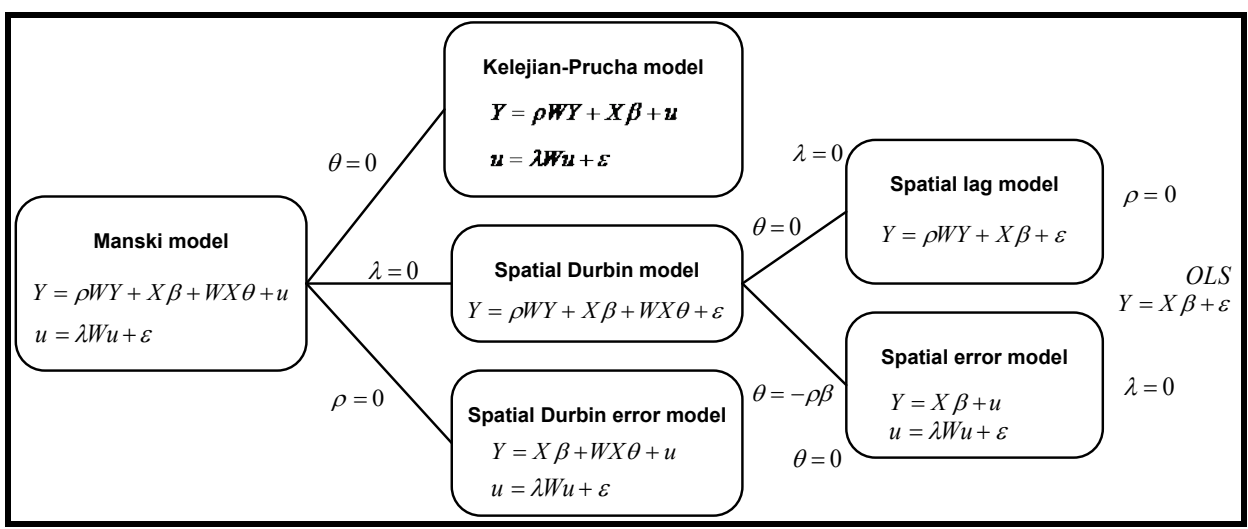

Fuente: Elhorst (2010).

Una vez analizadas las posibles especificaciones de los modelos el siguiente paso ha sido la selección del modelo más adecuado al proceso generador de datos. Durante los últimos veinte años, el proceso habitual era una estrategia que iba de lo particular a lo general, en concreto, mayoritariamente se utiliza como referencia estándar el trabajo de Florax et al. (2003) donde a partir de la estimación mediante OLS se diseña una estrategia de selección en función de los resultados de los test LM y LM robustos (Anselin et al., 1996). Sin embargo, recientemente, autores como Mur y Angulo (2009) ponen en duda la ventaja de esta estrategia y abogan por una selección de lo general a lo particular que, en algunos casos, puede ofrecer mejores resultados. En este capítulo, se ha utilizado la propuesta de Elhorst (2010) que es una combinación de ambas estrategias.

En primer lugar, se estima el modelo mediante OLS y, a continuación, se contrasta mediante los test LM anteriormente mencionados cuál es el modelo más adecuado, el del retardo espacial o el del error espacial. Estos contrastes se basan en los residuos del modelo OLS y siguen una distribución chi-cuadrado con un grado de libertad. El punto diferencial de la estrategia ocurre cuando el modelo OLS es rechazado a favor del lag, error o de ambos. Cuando sucede esto, el spatial Durbin model debe ser el modelo a estimar. Una vez estimado el spatial Durbin model se contrasta si es factible utilizar versiones simplificadas 
del mismo, es decir, el lag o error, mediante el test LR (likelihood ratio) cuyo cálculo es factible si el modelo es estimado mediante Máxima Verosimilitud (LM).

Las hipótesis a contrastar son las siguientes: $H_{o}: \theta=0$ y $H_{o}: \theta=-\rho \beta$. Ambos contrastes siguen una distribución chi-cuadrado con $k$ grados de libertad. Elhorst (2010) propone estimar el spatial Durbin model en el caso de que los contrastes LM (ó LM robustos) y LR apunten soluciones diferentes.

\subsection{Resultados}

Los resultados de las estimaciones que se presentan a continuación son una selección de las estimaciones realizadas. Las dos variables finalmente incluidas, desglosadas ambas por sexo, son la tasa de actividad procedente de la Encuesta de Población Activa y los salarios medios obtenidos a partir de las estadísticas de la Agencia Tributaria. En esta última variable se ha optado por utilizar el valor medio del salario de cada provincia en referencia al salario medio de España, es decir, se trata de un índice de salarios tomando como referencia el salario medio de España.

En primer lugar, se ha estimado el modelo mediante OLS calculándose los contrastes LM y LM robustos si bien previamente se ha contrastado la normalidad de la perturbación aleatoria tal y como se recoge en las Tablas 4,5 y $6^{4}$. Los resultados de estos contrastes se incluyen en la En las Tablas 4, 5 y 6 se recogen los principales resultados de los modelos estimados mediante OLS, el de retardo espacial y el del error espacial, aunque tal y como se ha demostrado previamente, el modelo del retardo espacial es el que ofrece mayor fiabilidad.

Tabla 3. Los resultados del test LM indican que el modelo más adecuado es el modelo del retardo espacial, con la única excepción del peso del SPE para los hombres en el año 2009. Los resultados del test LR que comparan la especificación del modelo Durbin con el modelo lag y el error, penúltima y última fila de la tabla respectivamente, no son significativos indicando que no pueden rechazarse las hipótesis establecidas y, por lo tanto, no existen razones para descartar el modelo del retardo espacial a favor del spatial Durbin model ni, por lo tanto, descartar el modelo del error espacial a favor del spatial Durbin error model, en este último caso con la excepción de las mujeres. No obstante, en el caso de las mujeres el spatial lag siempre es claramente superior al spatial error.

En las Tablas 4, 5 y 6 se recogen los principales resultados de los modelos estimados mediante OLS, el de retardo espacial y el del error espacial, aunque

\footnotetext{
${ }^{4}$ Para un análisis detallado sobre los diferentes métodos de estimación y los contrastes de autocorrelación y heterogeneidad espacial véase Chasco (2003).
} 
tal y como se ha demostrado previamente, el modelo del retardo espacial es el que ofrece mayor fiabilidad.

Tabla 3

Selección de modelos: contrastes LM, LM robustos y LR

\begin{tabular}{|c|c|c|c|c|c|c|c|c|c|}
\hline & \multicolumn{3}{|c|}{2007} & \multicolumn{3}{|c|}{2008} & \multicolumn{3}{|c|}{2009} \\
\hline & $\begin{array}{l}\text { Ambos } \\
\text { sexos }\end{array}$ & Hombres & Mujeres & $\begin{array}{c}\text { Ambos } \\
\text { sexo }\end{array}$ & Hombres & Mujeres & $\begin{array}{l}\text { Ambos } \\
\text { sexos }\end{array}$ & Hombres & Mujeres \\
\hline Lmlag & $4,187^{* * *}$ & $3,085^{\star}$ & $8,464^{* * *}$ & $4,827^{\star *}$ & $8,871^{* * *}$ & 2,468 & $8,580^{* * *}$ & $7,638^{* * *}$ & $10,347^{\star * *}$ \\
\hline RLMlag & $3,792^{*}$ & 1,363 & $6,931^{\text {*** }}$ & $2,844^{*}$ & $8,290^{\star \star *}$ & 0,323 & 1,736 & 0,000 & $7,552^{\star \star \star}$ \\
\hline Lmerr & 1,250 & 1,733 & $3,184^{*}$ & 2,134 & 2,479 & 2,458 & $7,101^{\text {***}}$ & $10,291^{* * *}$ & $3,957^{\star *}$ \\
\hline RLMerr & 0,854 & 0,011 & 1,652 & 0,150 & 1,898 & 0,313 & 0,257 & 2,653 & 1,162 \\
\hline$H_{o}: \theta=0$ & 3,135 & 0,165 & 2,092 & 2,379 & 0,421 & 2,238 & 3,299 & 2,017 & 2,124 \\
\hline$H_{o}: \theta=-\rho \beta$ & 3,743 & 1,548 & $6,078^{* *}$ & 2,937 & 0,7836 & $7,510^{* *}$ & 2,607 & 0,637 & $6,483^{* *}$ \\
\hline
\end{tabular}

Nota: * significativo al 10\%, ${ }^{* *}$ significativo al $5 \%$, ${ }^{* * *}$ significativo al $1 \%$.

Fuente: Elaboración propia.

Tabla 4

Principales resultados de los modelos desglosados por sexo, 2007

\begin{tabular}{|c|c|c|c|c|c|c|c|c|c|}
\hline & \multicolumn{3}{|c|}{ MCO } & \multicolumn{3}{|c|}{ Modelo del retardo espacial } & \multicolumn{3}{|c|}{ Modelo del error espacial } \\
\hline & $\begin{array}{c}\text { Ambos } \\
\text { sexos }\end{array}$ & Hombres & Mujeres & $\begin{array}{l}\text { Ambos } \\
\text { Sexos }\end{array}$ & Hombres & Mujeres & $\begin{array}{c}\text { Ambos } \\
\text { sexos }\end{array}$ & Hombres & Mujeres \\
\hline Tasa de actividad & $\begin{array}{c}-4,696 \\
(1,169)^{\star \star *}\end{array}$ & - & - & $\begin{array}{c}-4,695 \\
(1,081)^{\star \star \star}\end{array}$ & - & - & $\begin{array}{c}-4,418 \\
(1,217)^{\star \star \star *} \\
\end{array}$ & - & - \\
\hline T. Act. hombres & - & $\begin{array}{c}-5,654 \\
(1,160)^{\star * *}\end{array}$ & - & - & $\begin{array}{c}-5,034 \\
(1,133)^{\star \star *}\end{array}$ & - & - & $\begin{array}{c}-5,414 \\
(1,212)^{\star * *}\end{array}$ & - \\
\hline T. Act. mujeres & - & - & $\begin{array}{c}-3,607 \\
(1,098)^{\star * *} \\
\end{array}$ & - & - & \begin{tabular}{|c|}
$-3,165$ \\
$(0,945)^{\star \star \star}$ \\
\end{tabular} & - & - & $\begin{array}{c}-3,028 \\
(1,115)^{\star \star \star}\end{array}$ \\
\hline Salario & $\begin{array}{c}-2,370 \\
(0,873)^{\star * \star}\end{array}$ & - & - & $\begin{array}{c}-1,557 \\
(0,772)^{\star *}\end{array}$ & - & - & $\begin{array}{c}-2,173 \\
(0,948)^{\star \star} \\
\end{array}$ & - & - \\
\hline Salario hombres & - & $\begin{array}{c}-1,702 \\
(0,703)^{\star * \star}\end{array}$ & - & - & $\begin{array}{c}-1,705 \\
(0,652)^{\star * *}\end{array}$ & - & - & $\begin{array}{c}-1,695 \\
(0,732)^{\star *} \\
\end{array}$ & - \\
\hline Salario mujeres & - & - & $\begin{array}{c}-2,839 \\
(1,040)^{\star * *}\end{array}$ & - & - & $\begin{array}{c}-1,659 \\
(0,926)^{\star *}\end{array}$ & - & 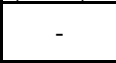 & $\begin{array}{c}-1,947 \\
(1,313) \\
\end{array}$ \\
\hline Constante & $\begin{array}{c}-6,239 \\
(0,662)^{\star * *}\end{array}$ & $\begin{array}{c}-5,971 \\
(0,467)^{\star * *}\end{array}$ & $\begin{array}{c}-6,204 \\
(0,843)^{\star * \star}\end{array}$ & $\begin{array}{c}-4,955 \\
(0,932)^{\star \star \star}\end{array}$ & $\begin{array}{c}-4,654 \\
(0,852)^{\star * *}\end{array}$ & $\begin{array}{c}-4,117 \\
(0,909)^{* * \star}\end{array}$ & $\begin{array}{c}-6,064 \\
(0,687)^{\star * *} \\
\end{array}$ & $\begin{array}{c}-5,879 \\
(0,488)^{\star * *}\end{array}$ & $\begin{array}{c}-5,675 \\
(0,854)^{\star * *}\end{array}$ \\
\hline$\rho$ & $1-1,1$ & 1 & $x_{1}=1$ & $\begin{array}{c}0,367 \\
(0,163)^{\star *}\end{array}$ & $\begin{array}{c}0,306 \\
(0,166)^{\star *}\end{array}$ & $\begin{array}{c}0,525 \\
(0,141)^{\star \star \star *} \\
\end{array}$ & 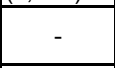 & $x=1$ & 中-1 \\
\hline$\lambda$ & - & - & - & - & - & - & $\begin{array}{c}0,275 \\
(0,218)\end{array}$ & $\begin{array}{c}0,263 \\
(0,220)\end{array}$ & $\begin{array}{c}0,504 \\
(0,170)^{\star * *}\end{array}$ \\
\hline Test LR & - & - & - & $3,680^{* *}$ & $2,768^{*}$ & $7,963^{* * *}$ & 1,239 & 1,385 & $3,977^{* *}$ \\
\hline Log likelihood & - & - & - & $-38,425$ & $-32,579$ & $-44,011$ & $-38,739$ & $-33,270$ & $-46,004$ \\
\hline AIC & - & - & - & 86,851 & 75,159 & 98,023 & 87,479 & 76,542 & 102,010 \\
\hline Test LM & - & - & - & 0,549 & 0,058 & 0,268 & - & - & - \\
\hline \begin{tabular}{|l} 
Test \\
Breusch-Pagan
\end{tabular} & - & - & - & 2,637 & 2,201 & 1,825 & 1,902 & 2,453 & 2,627 \\
\hline Jarque Bera & 1,111 & 0,164 & 1,110 & 0,083 & 0,192 & 0,005 & 0,759 & 0,057 & 0,452 \\
\hline
\end{tabular}

Nota: * significativo al 10\%, ** significativo al $5 \%$, ${ }^{* * *}$ significativo al $1 \%$.

Fuente: Elaboración propia. 


\section{Tabla 5}

Principales resultados de los modelos desglosados por sexo, 2008

\begin{tabular}{|c|c|c|c|c|c|c|c|c|c|}
\hline & \multicolumn{3}{|c|}{ MCO } & \multicolumn{3}{|c|}{ Modelo del retardo espacial } & \multicolumn{3}{|c|}{ Modelo del error espacial } \\
\hline & $\begin{array}{l}\text { Ambos } \\
\text { sexos }\end{array}$ & Hombres & Mujeres & $\begin{array}{c}\text { Ambos } \\
\text { Sexos }\end{array}$ & Hombres & Mujeres & $\begin{array}{l}\text { Ambos } \\
\text { sexos }\end{array}$ & Hombres & Mujeres \\
\hline Tasa de actividad & $\begin{array}{c}-4,103 \\
(0,944)^{\star \star \star}\end{array}$ & - & - & $\begin{array}{c}-3,907 \\
(0,878)^{\star \star \star}\end{array}$ & - & - & $\begin{array}{c}-3,787 \\
(1,013)^{\star * *}\end{array}$ & - & - \\
\hline T. Act. hombres & - & $\begin{array}{c}-4,789 \\
(0,928)^{\star \star \star *}\end{array}$ & - & - & $\begin{array}{c}-4,206 \\
(0,914)^{\star * *}\end{array}$ & - & 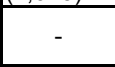 & $\begin{array}{c}-4,481 \\
(0,987)^{\star * *}\end{array}$ & - \\
\hline T. Act. mujeres & - & (1), & $\begin{array}{c}-3,513 \\
(0,965)^{* * *}\end{array}$ & - & ק & \begin{tabular}{|c|}
$-3,094$ \\
$(0,820)^{* * *}$
\end{tabular} & - & ( & $\begin{array}{c}-2,991 \\
(1,019)^{\star * \star}\end{array}$ \\
\hline Salario & $\begin{array}{c}-2,336 \\
(0,718)^{\star * *}\end{array}$ & - & - & $\begin{array}{c}-1,709 \\
(0,665)^{\star *}\end{array}$ & - & - & $\begin{array}{c}-2,188 \\
(0,809)^{\star * * *}\end{array}$ & - & - \\
\hline Salario hombres & (0, & $\begin{array}{c}-1,678 \\
(0,600)^{\star \star * *}\end{array}$ & - & - & $\begin{array}{c}-1,740 \\
(0,559)^{\star * \star}\end{array}$ & - & - & $\begin{array}{c}-1,901 \\
(0,635)^{\star * *}\end{array}$ & - \\
\hline Salario mujeres & - & - & $\begin{array}{c}-2,805 \\
(0,908)^{\star * *}\end{array}$ & - & - & $\begin{array}{c}-1,621 \\
(0,798)^{\star *}\end{array}$ & - & - & $\begin{array}{c}-1,890 \\
(1,167)\end{array}$ \\
\hline Constante & $\begin{array}{c}-5,786 \\
(0,518)^{\star * *}\end{array}$ & $\begin{array}{c}-5,545 \\
(0,376)^{\star \star *}\end{array}$ & $\begin{array}{c}-5,981 \\
(0,694)^{\star * *}\end{array}$ & $\begin{array}{c}-4,305 \\
(0,786)^{\star \star \star}\end{array}$ & $\begin{array}{c}-4,217 \\
(0,764)^{\star * \star}\end{array}$ & $\begin{array}{c}-3,867 \\
(0,775)^{\star * \star}\end{array}$ & $\begin{array}{c}-5,603 \\
(0,554)^{\star * *}\end{array}$ & $\begin{array}{c}-5,454 \\
(0,402)^{\star * *}\end{array}$ & $\begin{array}{c}-5,513 \\
(0,730)^{\star * *}\end{array}$ \\
\hline$\rho$ & - & - & - & $\begin{array}{c}0,405 \\
(0,148)^{\star \star \star}\end{array}$ & $\begin{array}{c}0,323 \\
(0,159)^{\star *}\end{array}$ & $\mid \begin{array}{c}0,546 \\
(0,130)^{\star * \star}\end{array}$ & - & - & - \\
\hline$\lambda$ & - & - & - & (10, & $1(0,1004$ & - & $\begin{array}{c}0,379 \\
(0,198)^{* *}\end{array}$ & $\begin{array}{c}0,358 \\
(0,202)^{*}\end{array}$ & $\begin{array}{c}0,529 \\
(0,164)^{\star * \star}\end{array}$ \\
\hline Test LR & - & - & - & $4,398^{\star *}$ & $2,679^{*}$ & $9,062^{* * *}$ & 2,287 & 2,317 & $3,790^{* *}$ \\
\hline Log likelihood & - & - & - & $-31,571$ & $-28,303$ & $-37,172$ & $-31,850$ & $-28,484$ & $-39,808$ \\
\hline AIC & - & - & - & 73,142 & 66,606 & 84,345 & 73,701 & 66,968 & 89,617 \\
\hline Test LM & - & - & - & 0,014 & 0,672 & 0,095 & - & - & - \\
\hline $\begin{array}{l}\text { Test } \\
\text { Breusch-Pagan }\end{array}$ & - & - & - & 3,177 & 1,440 & 4,155 & 1,940 & 2,082 & 3,825 \\
\hline Jarque Bera & 0,316 & 1,140 & 0,212 & 0,253 & 0,790 & 0,507 & 0,113 & 0,572 & 0,413 \\
\hline
\end{tabular}

Nota: ${ }^{*}$ significativo al $10 \%,{ }^{* *}$ significativo al $5 \%$, ${ }^{* * *}$ significativo al $1 \%$.

Fuente: Elaboración propia.

En todos los casos el parámetro espacial $\rho$ es significativo y las estimaciones obtenidas son mayores en el caso de las mujeres, con valores superiores a 0,50 , mientras que en el caso de los hombres oscila entre 0,30 en 2007 y 0,49 en 2009. El incremento del peso de las colocaciones del SPE sobre las de mercado en una determinada provincia da lugar a que se extienda ese mismo fenómeno hacia el resto de provincias pertenecientes a un mismo SPE autonómico.

En todos los casos los modelos estimados corrigen la autocorrelación espacial tal y como indica el test LM. Además, el test de Breusch-Pagan indica que los modelos no muestran problemas de heterocedasticidad excepto en el último modelo (mujeres, año 2009).

La variable tasa de actividad es significativa y negativa en todos los casos. La relación negativa indica cómo en aquellas provincias con una mayor tasa de actividad, el peso de las colocaciones del SPE sobre las colocaciones de mercado es menor. Una mayor tasa de actividad está asociada a un mejor funcionamiento del mercado de trabajo en términos de colocaciones, por lo que la actividad del SPE es menor. La incidencia de la tasa de actividad es mayor en el caso de los hombres que en el de las mujeres si bien es cierto que el diferencial 
de ambos coeficientes disminuye a lo largo de 2008 y en mayor medida en 2009, un resultado acorde al funcionamiento del mercado de trabajo. La variable salarios también es significativa y negativa en todos los casos, siendo mayor el efecto para los hombres que para las mujeres en lo que respecta a la importancia del SPE en el mercado de trabajo. Esta relación negativa no es sorprendente puesto que un mayor salario medio refleja un mayor nivel de productividad en la provincia correspondiente y, por lo tanto, un mejor comportamiento económico de la misma. Esto se traduce en un mayor dinamismo del mercado de trabajo siendo menos necesaria la participación del SPE en el proceso de emparejamiento de vacantes y trabajadores.

\section{Tabla 6}

Principales resultados de los modelos desglosados por sexo, 2009

\begin{tabular}{|c|c|c|c|c|c|c|c|c|c|}
\hline & \multicolumn{3}{|c|}{ MCO } & \multicolumn{3}{|c|}{ Modelo del retardo espacial } & \multicolumn{3}{|c|}{ Modelo del error espacial } \\
\hline & $\begin{array}{l}\text { Ambos } \\
\text { sexos }\end{array}$ & Hombres & Mujeres & $\begin{array}{l}\text { Ambos } \\
\text { Sexos }\end{array}$ & Hombres & Mujeres & $\begin{array}{l}\text { Ambos } \\
\text { sexos }\end{array}$ & Hombres & Mujeres \\
\hline Tasa de actividad & $\begin{array}{c}-4,176 \\
(1,028)^{\star \star \star}\end{array}$ & - & - & $\begin{array}{c}-4,204 \\
(0,921)^{\star \star \star}\end{array}$ & - & - & $\begin{array}{c}-4,519 \\
(1,103)^{\star \star *}\end{array}$ & - & - \\
\hline T. Act. hombres & - & $\begin{array}{c}-4,187 \\
(1,150)^{\star \star *}\end{array}$ & - & - & $\begin{array}{c}-3,853 \\
(1,036)^{\star * *}\end{array}$ & - & - & $\begin{array}{c}-4,760 \\
(1,250)^{\star * *}\end{array}$ & - \\
\hline T. Act. mujeres & - & - & $\begin{array}{c}-3,804 \\
(0,999)^{\star * *}\end{array}$ & - & - & $\begin{array}{c}-3,441 \\
(0,841)^{\star * \star}\end{array}$ & - & - & $\begin{array}{c}-3,695 \\
(1,018)^{\star \star \star}\end{array}$ \\
\hline Salario & $\begin{array}{c}-2,501 \\
(0,696)^{* * *}\end{array}$ & - & - & $\begin{array}{c}-1,742 \\
(0,615)^{\star * *}\end{array}$ & - & - & $\begin{array}{c}-1,743 \\
(0,817)^{\star *}\end{array}$ & - & - \\
\hline Salario hombres & - & $\begin{array}{c}-1,809 \\
(0,660)^{\star * *}\end{array}$ & - & - & $\begin{array}{c}-1,720 \\
(0,576)^{\star * \star}\end{array}$ & - & - & $\begin{array}{c}-1,544 \\
(0,724)^{* * *}\end{array}$ & - \\
\hline Salario mujeres & - & - & $\begin{array}{c}-2,970 \\
(2,970)^{\star * \star}\end{array}$ & - & - & $\begin{array}{c}-1,721 \\
(0,710)^{\star *}\end{array}$ & - & - & $\begin{array}{l}-1,342 \\
(1,051)\end{array}$ \\
\hline Constante & $\begin{array}{c}-5,553 \\
(0,578)^{\star \star *}\end{array}$ & $\begin{array}{c}-4,909 \\
(0,488)^{\star * \star}\end{array}$ & $\begin{array}{c}-6,011 \\
(0,724)^{\star * *}\end{array}$ & $\begin{array}{c}-4,041 \\
(0,746)^{\star * \star}\end{array}$ & $\begin{array}{c}-3,325 \\
(0,695)^{\star * *}\end{array}$ & $\begin{array}{c}-3,958 \\
(0,780)^{* * *}\end{array}$ & $\begin{array}{c}-5,708 \\
(0,617)^{\star * *}\end{array}$ & $\begin{array}{c}-5,175 \\
(0,530)^{\star * *}\end{array}$ & $\begin{array}{c}-5,794 \\
(0,741)^{\star \star \star}\end{array}$ \\
\hline$\rho$ & - & - & - & $\begin{array}{c}0,500 \\
(0,133)^{\star * \star}\end{array}$ & $\begin{array}{c}0,495 \\
(0,144)^{\star * *}\end{array}$ & $\begin{array}{c}0,571 \\
(0,124)^{* * *}\end{array}$ & - & - & - \\
\hline$\lambda$ & - & - & - & - & - & - & $\begin{array}{c}0,561 \\
(0,156)^{\star \star \star *}\end{array}$ & $\begin{array}{c}0,570 \\
(0,154)^{\star \star \star *}\end{array}$ & $\begin{array}{c}0,613 \\
(0,142)^{\star \star *}\end{array}$ \\
\hline Test LR & - & - & - & $7,473^{* * *}$ & $6,881^{\star \star *}$ & $10,273^{* * *}$ & $6,711^{* * *}$ & $8,261^{* * *}$ & $5,914^{* *}$ \\
\hline Log likelihood & - & - & - & $-30,876$ & $-31,113$ & $-36,325$ & $-30,530$ & $-30,423$ & $-38,505$ \\
\hline AIC & - & - & - & 71,753 & 72,227 & 82,652 & 71,061 & 70,847 & 87,011 \\
\hline Test LM & - & - & - & 1,093 & $3,232^{*}$ & 0,002 & - & - & - \\
\hline \begin{tabular}{|l} 
Test \\
Breusch-Pagan
\end{tabular} & - & - & - & 3,757 & 1,290 & $6,692^{* *}$ & 4,033 & 3,436 & $6,414^{\star *}$ \\
\hline Jarque Bera & 0,260 & 0,660 & 0,176 & 0,413 & 1,896 & 0,159 & 0,810 & 1,080 & 0,256 \\
\hline
\end{tabular}

Nota: * significativo al 10\%, ** significativo al $5 \%$, ${ }^{* * *}$ significativo al $1 \%$.

Fuente: Elaboración propia. 


\section{CONCLUSIONES Y RECOMENDACIONES}

En este artículo se analiza la eficiencia del Servicio Público de Empleo tomando como referencia la cuota de mercado del mismo a nivel provincial. Para ello, se ha calculado el peso de las colocaciones del SPE sobre las colocaciones de mercado a nivel provincial. Los resultados obtenidos muestran que la estructura espacial de la distribución de colocaciones en España es bastante compleja. El análisis realizado proporciona una primera aproximación a esta distribución en los años 2007, 2008 y 2009.

En base a los "box-map" se observa que existen importantes disparidades a nivel provincial. La presencia del SPE en el mercado de trabajo es mayor en la parte sur de España, principalmente en Andalucía, Castilla-La Mancha y Extremadura (especialmente para las mujeres) mientras que es más limitada en la zona mediterránea.

Además, se justifica la necesidad de incluir en la especificación de los modelos la presencia de dependencia espacial, los valores de las colocaciones del SPE sobre las colocaciones de mercado no son independientes en el espacio. A pesar de la escasez de estudios de carácter empírico sobre la dinámica espacial del mercado de trabajo en España, la existencia de dependencia espacial está ampliamente contrastada en estudios internacionales. Para recoger la multidireccionalidad que caracteriza las relaciones espaciales se ha definido, en primer lugar, una matriz de pesos espaciales en la que se recoge tanto el clásico criterio de contigüidad física como el hecho de que varias provincias comparten un mismo modelo de gestión del SPE autonómico.

Respecto a la metodología, después de estudiar cuál es el modelo espacial más apropiado para explicar la cuota de mercado del SPE se ha optado por el modelo del retardo espacial por ser éste el que mejores resultados muestra en los contrastes de selección del modelo. Está claro que la mayor o menor presencia del SPE en el mercado de trabajo depende fundamentalmente del comportamiento del mercado de trabajo en sí mismo. Dos variables que tratan de aproximar este hecho y que se han incluido en el modelo final son la tasa de actividad y el salario medio.

La relación entre la tasa de actividad y el peso de las colocaciones del SPE en el mercado de trabajo es negativa, tal y como cabe esperar a priori y de acuerdo a los resultados encontrados en otros países europeos. En el mismo sentido la variable salarios medios es significativa y negativa. Dado el patrón espacial diferencial para hombres y mujeres se ha estimado un modelo para cada colectivo siendo los efectos de ambas variables explicativas mayores para los hombres si bien es cierto que se atenúan a lo largo del tiempo, conforme empeora el ciclo económico. 
La distribución en el espacio de las colocaciones en las que interviene el SPE es totalmente diferente a la distribución de las colocaciones de mercado presentándose la primera más organizada espacialmente. Este resultado es coincidente con la hipótesis, ampliamente contrastada, de segmentación espacial del mercado de trabajo en España. La actuación del SPE es mayor en aquellos lugares en los que el mercado de trabajo muestra un menor dinamismo. Este resultado es debido a que el SPE, como institución, está presente en todo el territorio, de manera que las empresas pueden acudir al mismo cuando deseen, o cuando tengan dificultades para encontrar a los trabajadores que buscan, y que las oficinas de empleo gestionen sus puestos de trabajo. Su existencia contrarresta, parcialmente, los efectos derivados de las economías de aglomeración que hace que ciertas provincias atraigan un mayor número de empresas y trabajadores en busca de empleo.

\section{REFERENCIAS BIBLIOGRÁFICAS}

ALTHIN, R. y BEHRENZ, L. (2004). "An efficiency of Swedish Employment Offices". International Review of Applied Economics, 18(4), pp. 471-482.

ANSELIN, L. (1988). Spatial econometrics methods and models. Dordrecht: Kluwer Academic Publishers, The Netherlands.

ANSELIN, L.; BERA, A.; FLORAX, R. y YOON, M. (1996). "Simple diagnostic test for spatial dependence". Regional Science and Urban Economics, 26, pp. 77-104.

CHASCO, C. (2003). Econometría especial aplicada a la predicción-extrapolación de datos microterritoriales. Consejería de Economía e Innovación Tecnológica. Madrid.

CLIFF, A. y ORD, J.K. (1981). Spatial processes: models and applications. Pion, London.

CLINCH, J.P. y O'NEILL, E. (2009). "Applying spatial economics to national spatial planning". Regional Studies, 43(2), pp. 157-178.

DE KONING, J.; DENYS, J. y WALWEI, U. (1999). "Deregulation in placement services: a comparative study for eight EU countries." European Commission.

FERTIG, M.; SCHMIDT, C.M. y SCHNEIDER, H. (2006). "Active labor market policy in Germany-ls there a successful policy strategy?" Regional Science and Urban Economics, 36, pp. 399-430.

JIMENO, J.F. (1993). "La reforma del Instituto Nacional de Empleo como mecanismo de intermediación en el mercado de trabajo". Boletín del Círculo de Empresarios, 57, pp. 235-252. 
ELHORST, J.P. (2010). "Applied Spatial Econometrics: Raising the BAR". Spatial Economic Analysis, 5(1), pp. 9-28.

ELHORST, J.P. (2008). "A spatiotemporal analysis of aggregate labour force behaviour by sex and age across the European Union". Journal of Geographical Systems, 10, pp. 167-190.

ELHORST, J.P. (2003). "The mystery of regional unemployment differentials: theoretical and empirical explanations". Journal of Economic Surveys, 17, pp. 709-748.

ELHORST, J.P. y ZEILSTRA, A.S. (2007). "Labour force participation rates at the regional and national levels of the European Union: an integrated analysis". Papers in Regional Science, 86(4), pp. 525-549.

FLORAX, R.; FOLMER, H. y REY, S. (2003). "Specification searches in spatial econometrics: the relevance of Hendry's methodology". Regional Science and Urban Economics, 33, pp. 557-579.

KARLSSON, C. y HAYINES, K. (2002). "Regional labour markets in transition". Papers in Regional Science, 81, pp. 301-304.

LESAGE, J. y PACE, R.K. (2009). Introduction to Spatial Econometric. CRC Press.

LONGHI, S. y NIJKAMP, P. (2007). "Forecasting regional labor market developments under spatial autocorrelation". International Regional Science Review, 30(2), pp. 110-119.

LÓPEZ-BAZO, E.; DEL BARRIO, T. y ARTIS, M. (2005). "Geographical distribution of unemployment in Spain”. Regional Studies, 39(3), pp. 305-318.

LÓPEZ-BAZO, E., DEL BARRIO, T. y ARTIS, M. (2002). "The regional distribution of Spanish unemployment: a spatial analysis". Papers in Regional Science, 81, pp. 365-389.

MANSKI, C.F. (1993). "Identification of endogenous social effects: the reflection problem". Review of Economic Studies, 60, pp. 531-542.

MAYOR, M. y LOPEZ, A.J. (2008). "Spatial shift-share analysis versus spatial filtering: an application to Spanish employment data". Empirical Economics, 34(1), pp. 123-142.

MOLHO, I. (1995). "Spatial autocorrelation in British unemployment". Journal of Regional Science, 35(4), pp. 641-658.

MUR, J. y ANGULO, A. (2009). "Model selection strategies in a spatial setting: Some

additional results". Regional Science and Urban Economics, 39, pp. 200-213.

OVERMAN, H.G. y PUGA, D. (2002). "Unemployment clusters across Europe's regions and countries". Economic Policy, 17(34), pp. 117-147.

PAELINCK, J.H.P. y KLAASSEN, L.H. (1979). Spatial Econometrics. Saxon House: Farnborough.

PATACCHINI, E. y ZENOU, Y. (2007). "Spatial dependence in local unemployment rates". Journal of Economic Geography, 7, pp. 169-191. 
RUSSO, G.; GORTER, C. y SCHETTKAT, R. (2001). "Searching, hiring and labour market conditions". Labour Economics, 8, pp. 553-571.

SHELDON, G.M. (2003). "The efficiency of Public Employment Services: a nonparametric matching function analysis for Switzerland". Journal of Productivity Analysis, 20, pp. 49-70.

SUÁREZ, P.; MAYOR, M.; CUETO, B. (2012). "The accessibility to employment offices in the Spanish labour market". Papers in Regional Science. DOI: 10.1111/j.1435-5957.2012.00425.x.

SUÁREZ, P.; MAYOR, M. (2012). "La intermediación laboral del Servicio Público de Empleo en España: un análisis regional con los datos del SISPE". Revista de Ministerio de Trabajo e Inmigración, Serie Economía y Sociología, 96, pp. 175-194.

SUÁREZ, P.; MAYOR, M.; CUETO, B. (2011). "How important is access to employment offices in Spain? An urban and non-urban perspective". Investigaciones regionales, 21, pp. 119-140.

SUÁREZ, P. y MAYOR, M. (2009). "Spatial analysis of public employment services in the Spanish provinces". Documento de Trabajo FUNCAS, $n^{\circ} 485$.

TOHARIA, L. (2007). Las estadísticas administrativas como fuentes de información para el estudio del mercado de trabajo andaluz. Instituto de Estadística de Andalucía, Consejería de Economía y Hacienda.

VASSILIEV, A.; FERRO-LUZZI, G.; FLÜCKIGER, Y. y RAMIREZ, J.V. (2006). "Unemployment and employment offices' efficiency: what can be done?" Socio-Economic Planning Sciences, 40, pp. 169-186. 